\title{
Combining Mendonça-Cipolla Self-calibration and Scene Constraints
}

\author{
Adlane Habed $^{1}$, Tarik Elamsy ${ }^{2}$, and Boubakeur Boufama ${ }^{2}$ \\ 1 Université de Bourgogne \\ Laboratoire Le2i, UMR CNRS 5158 \\ BP16, Route des plaines de l'Yonne \\ 89010 Auxerre Cedex, France \\ adlane.habed@u-bourgogne.fr \\ 2 University of Windsor \\ School of Computer Science \\ 401 Sunset Avenue \\ Windsor, ON. N9B 3P4, Canada \\ \{elamsy, boufama\}@uwindsor.ca
}

\begin{abstract}
In this paper, we propose a method that combines plane parallelism and the Mendonça/Cipolla self-calibration constraints. In our method each pair of images is treated independently and can therefore use a different pair of parallel planes not necessarily visible in the other views. While, for each pair of images, constraints on the singular values of the essential matrix provide two algebraic constraints on the intrinsic parameters, those we derive from plane parallelism have the advantage of providing two additional ones making the calibration of a no-skew camera possible from two images only.
\end{abstract}

Keywords: self-calibration, 3D reconstruction, scene constraints.

\section{Introduction}

The problem of self-calibrating a camera, i.e. retrieving its intrinsic parameters solely from point correspondences across images, has been extensively addressed in the literature. It is both highly desirable and important that camera selfcalibration methods not only should they exhibit nice convergence, stability, robustness and accuracy properties but also have the ability to easily incorporate scene constraints such as parallelism and orthogonality whenever these are available. For instance, incorporating scene constraints in the self-calibration process may contribute in reducing the number of required images, obtaining more accurate $3 \mathrm{D}$ reconstructions and parameters as well as overcoming degeneracy issues generally due to special camera motions.

Vanishing points, possibly in conjunction with other constraints (such as the modulus constraints [8), are commonly used to help obtaining an estimate of the plane at infinity and hence recover the intrinsic parameters. Ideally, however, 
scene constraints need to be incorporated in the process of calculating the intrinsic parameters as to reduce degeneracy situations. These parameters are generally calculated through the recovery of the projective representations of either the so-called Image of the Absolute Conic (or its dual the DIAC), the Absolute Plane Quadric or the Absolute Line Quadric, all of which embed the internal geometry of the camera (the reader may refer to [3] and the reference therein for more details on the subject). The geometry of these conics and quadrics is well suited for expressing parallel and/or orthogonal directional constraints in a way these can be easily combined with self-calibration constraints 265. Quadricbased self-calibration methods require the prior calculation of camera matrices that are consistent with the same projective or quasi-affine [3] 3D reconstruction of the scene. These methods are known to perform better than their conic-based counterpart which are simpler, as they only require the epipolar geometry relating pairs images, but exhibit poor numerical stability and high sensitivity to both noise and initialization.

Also relying on the sole calculation of the epipolar geometry between pairs of views, Mendonça and Cipolla [7] have proposed an even simpler, yet effective, method which, unlike conic-based methods, exhibits remarkable convergence properties and recovers camera parameters with a fair accuracy [1]. Their method allows to directly estimate the camera parameters through constraints on the singular values of the essential matrix relating each pair of views. Despite the simplicity and the good performance of their self-calibration method, there has been, to our knowledge, no attempt to combine their constraints on the essential matrix and those from the scene.

In this paper, we propose a method that nicely combines plane parallelism and the Mendonça/Cipolla self-calibration constraints without explicitly referring to the geometry of neither the Image of the Absolute Conic nor its Dual. In our method, each pair of images is treated independently and can therefore use a different pair of parallel planes not necessarily visible in the other views. While, for each pair of images, constraints on the singular values of the essential matrix provide two constraints on the intrinsic parameters, those we derive from plane parallelism provide two additional ones making the calibration of a no-skew camera possible when using two images only. In addition, our experiments show that the proposed method significantly improves the quality of the estimated $3 \mathrm{D}$ reconstruction, that of the intrinsic parameters and the convergence of the algorithm in comparison with Mendonça/Cipolla self-calibration. Our results also show that this new method outperforms, in terms of accuracy of the 3D reconstruction, the recent stratified method presented in [2] which also exploits plane parallelism.

Our paper is organized as follows. A review of the Mendonça-Cipolla selfcalibration method is presented in Section 2, Our new constraints, which are due to plane parallelism, are derived in Section 3. A method for combining plane parallelism and the Mendonça and Cipolla self-calibration constraints is described in Section 4. The results of our experiments are detailed in Section 5 . Section 6 concludes our work. 


\section{Direct Self-calibration Constraints}

Assuming the cameras satisfy the pinhole model, it is well-known that two corresponding points $p_{i}$ and $p_{j}$ in two images $i$ and $j$ are related by the epipolar constraint: $p_{j}^{\top} F_{i j} p_{i}=0 . F_{i j}$ is a rank-2 $3 \times 3$ matrix known as the fundamental matrix while $\mathrm{p}_{\mathrm{i}}$ and $\mathrm{p}_{\mathrm{j}}$ are the homogeneous pixel coordinates of the projections, in the two images, of the same scene point. The fundamental matrix is closely related to the so-called essential matrix

$$
E_{i j}=\left[t_{j i}\right]_{\times} R_{i j}
$$

which embeds the translational $t_{j i}$ and the rotational $R_{i j}$ components of the rigid motion between the two cameras. Unlike the fundamental matrix, the essential matrix cannot be calculated from point correspondences across images unless the intrinsic parameters of both cameras are known [3]. These matrices are indeed related through

$$
E_{i j} \simeq A_{j}^{\top} F_{i j} A_{i}
$$

where the $A$ matrices are $3 \times 3$ upper-triangular and parameterized in terms of the pixel-valued focal lengths, $f_{u}$ and $f_{v}$, along the main axes of the image, the pixel coordinates $\left(u_{0}, v_{0}\right)$ of the principal point, and the skew factor $s$ of the camera under consideration; that is,

$$
\mathrm{A}=\left[\begin{array}{ccc}
f_{u} & s & u_{0} \\
0 & f_{v} & v_{0} \\
0 & 0 & 1
\end{array}\right]
$$

Mendonça and Cipolla's self-calibration method [7] is based on the property of the essential matrix having two equal singular values while the third one is zero. This property has been proved by Huang and Faugeras [4] who have also shown that this is a necessary and sufficient condition for a matrix to be factored into the product of an orthogonal and a skew-symmetric matrix. Moreover, it has been demonstrated that the equality of the singular values imposes two algebraic constraints on the essential matrix. Assuming the cameras have the same intrinsic parameters (e.g. a moving camera with constant parameters), the self-calibration method proposed in [7] consists in minimizing over $n \geq 3$ views the cost function

$$
\mathcal{C}(\mathrm{A})=\sum_{i j}^{n} \frac{w_{i j}}{\sum_{k l}^{n} w_{k l}} \frac{\sigma_{i j}^{+}-\sigma_{i j}^{-}}{\sigma_{i j}^{-}}
$$

where $\sigma_{i j}^{+}$and $\sigma_{i j}^{-}$are respectively the largest and the smallest non-zero singular values of $\mathrm{A}^{\top} \mathrm{F}_{\mathrm{ij}} \mathrm{A}$ and $w_{i j}$ are weights that are inversely proportional to a measurement of the quality of the calculated fundamental matrix.

\section{Scene Constraints}

We consider in the following a pair of parallel planes $\Pi$ and $\Phi$ observed by two cameras (from a sequence) whose fundamental matrix $F_{i j}$ and epipole $e_{j i}$ 
$\left(F_{i j}^{\top} e_{j i}=0\right)$ have already been recovered [3]. Being parallel, the two planes meet in a line on the plane at infinity. As a consequence, the $3 \mathrm{D}$ homogeneous coordinate vectors of our planes and that of the plane at infinity are linearly dependent. Such relationship is independent from the choice of the projective reference frame in which the coordinates of these planes are expressed. However, each pair of cameras, when considered independently from the others, allows to reconstruct the scene in a frame that is different from any frame chosen for some other pair of cameras. In order to emphasize this fact, we will denote by $\Pi_{\mathrm{ij}}, \Phi_{\mathrm{ij}}$ and $\infty_{\mathrm{ij}}$ the homogeneous coordinate vectors of $\Pi, \Phi$ and the plane at infinity, respectively, expressed in a projective frame chosen only for the pair of cameras $i$ and $j$ under consideration. Moreover, because plane coordinates are homogeneous and assuming none of the considered planes passes through the origin of the reference frame, we choose our plane coordinates scaled such that

$$
\Pi_{\mathrm{ij}}^{\top}=\left(\pi_{\mathrm{ij}}^{\top} 1\right), \Phi_{\mathrm{ij}}^{\top}=\left(\phi_{\mathrm{ij}}^{\top} 1\right) \text { and } \infty_{\mathrm{ij}}^{\top}=\left(\propto_{\mathrm{ij}}^{\top} 1\right) .
$$

In general, the linear dependency of these three planes is expressed through

$$
\infty_{\mathrm{ij}}=\alpha_{\mathrm{ij}} \Pi_{\mathrm{ij}}+\beta_{\mathrm{ij}} \Phi_{\mathrm{ij}}
$$

for some non-zero scalars $\alpha_{\mathrm{ij}}$ and $\beta_{\mathrm{ij}}$. However, for the choice of coordinates we have made in (5), these scalars must also verify $\alpha_{\mathrm{ij}}+\beta_{\mathrm{ij}}=1$. This allows to eliminate one of the scalars and thus further simplify the relationship between the directional components of the three planes; that is,

$$
\alpha_{i j}=\alpha_{i j} \pi_{i j}+\left(1-\alpha_{i j}\right) \phi_{i j} .
$$

Since we are free to choose the projective frame in which to express the scene and cameras, we might as well choose it such that the reference frame is attached to camera $i$ and the projection matrices of the two cameras given by $\mathrm{M}_{\mathbf{i}}=[\mathrm{I} \mid 0]$ and $M_{j}=\left[\left[e_{j i}\right]_{\times} F_{i j} \mid e_{j i}\right]$. This choice of matrices is often considered in the literature as a "starting" projective reconstruction 3 since it is fairly simple to obtain. In particular, it allows us to use only the epipolar geometry of the pair of cameras in order to retrieve the vectors $\pi_{\mathrm{ij}}$ and $\phi_{\mathrm{ij}}$ in the frame under consideration. For instance, if $\mathrm{p}_{\mathrm{i}}$ and $\mathrm{p}_{\mathrm{j}}$ are the homogeneous pixel coordinates of the projections in images $i$ and $j$ of the same $3 \mathrm{D}$ point $P$ on a plane $\Pi$, our choice of $\mathrm{M}_{\mathrm{i}}$ indicates that $\mathrm{P} \simeq\left(\mathrm{p}_{\mathrm{i}} \lambda_{\mathrm{i}}\right)$ for some value of the projective depth $\lambda_{i}$. Because $P$ lies on $\Pi$, the projective depth is given by $\lambda_{\mathrm{i}}=\pi_{\mathrm{ij}}^{\top} \mathrm{p}_{\mathrm{i}}$. Projecting $P$ on image $j$ via the projection matrix $M_{j}$ leads to the relationship $\mathrm{p}_{\mathrm{j}} \simeq\left(\left[\mathrm{e}_{\mathrm{ji}}\right]_{\times} \mathrm{F}_{\mathrm{ij}}+\mathrm{e}_{\mathrm{ji}} \pi_{\mathrm{ij}}^{\top}\right) \mathrm{p}_{\mathrm{i}}$. Since the latter relationship it true for any plane, the inter-image homographies induced by $\Pi$ and $\Phi$ are given by

$$
H_{i j \Pi} \simeq\left[e_{j i}\right]_{\times} F_{i j}+e_{j i} \pi_{i j}^{\top} \text { and } H_{i j \phi} \simeq\left[e_{j i}\right]_{\times} F_{i j}+e_{j i} \phi_{i j}^{\top}
$$

which allow the calculation of $\pi_{\mathrm{ij}}$ and $\phi_{\mathrm{ij}}$ from point correspondences assuming points on each plane are identified and their projections in the two matched images. Similarly, the inter-image homography of the plane at infinity will also be on the form

$$
\mathrm{H}_{\mathrm{ij} \infty} \simeq\left[\mathrm{e}_{\mathrm{ji}}\right]_{\times} \mathrm{F}_{\mathrm{ij}}+\mathrm{e}_{\mathrm{ji}} \propto_{\mathrm{ij}}^{\top}
$$


where $\propto_{i j}$ remains unknown. Using the relationship (7) that is due to plane parallelism, we can however reduce $\mathrm{H}_{\mathrm{ij} \infty}$ to the $1 \mathrm{D}$ family of matrices:

$$
\mathrm{H}_{\mathrm{ij} \infty} \simeq\left[\mathrm{e}_{\mathrm{ji}}\right]_{\times} \mathrm{F}_{\mathrm{ij}}+\alpha_{\mathrm{ij}} \mathrm{e}_{\mathrm{ji}} \pi_{\mathrm{ij}}^{\top}+\left(1-\alpha_{\mathrm{ij}}\right) \mathrm{e}_{\mathrm{ji}} \phi_{\mathrm{ij}}^{\top} .
$$

The product $\mathrm{H}_{\mathrm{ij} \infty}\left[\pi_{\mathrm{ji}}-\phi_{\mathrm{ij}}\right]_{\times}$eliminates the unknown scalar $\alpha_{\mathrm{ij}}$ from the above expression leading after simplification to

$$
\mathrm{H}_{\mathrm{ij} \infty}\left[\pi_{\mathrm{ij}}-\phi_{\mathrm{ij}}\right]_{\times} \simeq\left[\mathrm{e}_{\mathrm{ji}}\right]_{\times} \mathrm{F}_{\mathrm{ij}}\left[\pi_{\mathrm{ij}}-\phi_{\mathrm{ij}}\right]_{\times}-\mathrm{e}_{\mathrm{ji}} \pi_{\mathrm{ij}}^{\top}\left[\phi_{\mathrm{ij}}\right]_{\times} \cdot
$$

Moreover, because $H_{i j \infty}$ can be factored into $H_{i j \infty}=A_{j} R_{i j} A_{i}^{-1}$, one can easily deduce that, just like the essential matrix, $A_{j}^{-1} H_{i j \infty}\left[\pi_{i j}-\phi_{i j}\right]_{\times} A_{i}^{-\top}$ can be factored into the product $R_{i j}[q]_{\times}$of the rotation matrix $R_{i j}$ and a skew-symmetric matrix $[q]_{\times}=A_{i}^{-1}\left[\pi_{i j}-\phi_{i j}\right]_{\times} A_{i}^{-\top}$. As a result, the matrix $D_{i j \pi \phi}$ such that

$$
D_{i j \pi \phi}=A_{j}^{-1}\left(\left[e_{j i}\right]_{\times} F_{i j}\left[\pi_{i j}-\phi_{i j}\right]_{\times}-e_{j i} \pi_{i j}^{\top}\left[\phi_{i j}\right]_{\times}\right) A_{i}^{-\top}
$$

must have two equal singular values while the third one is zero. The existence of the zero singular value is by construction of the matrix $\mathrm{D}_{\mathrm{ij} \pi \phi}$. However, the equality of the other two singular values provides two additional algebraic constraints on the intrinsic parameters of the camera.

\section{Constraints Combination}

In general, when scene and self-calibration constraints are combined, the problem is cast into a constrained nonlinear optimization one. This is, for instance, the case in [5] where self-calibration and orthogonal planes constraints are combined. However, because the scene and all the cameras are reconstructed in the same frame, each pair of such planes provides only one constraint regardless of the length of the sequence. In the present paper, the situation is different since, in the absence of common frame for all cameras, the fact that two planes are parallel must be taken into account by every pair of images in which those planes are visible. Moreover, for each pair of images the constraints on $D_{\mathrm{ij} \pi \phi}$ are as important as those on the essential matrix since they introduce the same number of algebraic constraints. As a consequence, we have chosen to cast the problem of combining the constraints on the essential matrix $E_{i j}$ and those on $\mathrm{D}_{\mathrm{ij} \pi \phi}$ in an unconstrained optimization procedure. For $n \geq 2$ images captured by a moving camera (constant parameters), we propose the following cost function

$$
\mathcal{M}(\mathrm{A})=\sum_{i j}^{n} \frac{w_{i j}}{\sum_{k l}^{n} w_{k l}} \frac{\sigma_{i j}^{+}-\sigma_{i j}^{-}}{\sigma_{i j}^{-}}+\sum_{(\Pi, \Phi) \in \mathcal{S}} \gamma_{\Pi \Phi} \sum_{i j}^{n} \frac{v_{i j \Pi \Phi}}{\sum_{k l}^{n} v_{k l \Pi \Phi}} \frac{\delta_{i j \Pi \Phi}^{+}-\delta_{i j \Pi \Phi}^{-}}{\delta_{i j \Pi \Phi}^{-}}
$$

where $\mathcal{S}$ is the set of all pairs of identified parallel planes in the scene. The weights $v_{i j \Pi \Phi}$ are inversely proportional to the quality of the homography matrices (8) of the planes under consideration if the latter are visible in images $i$ and $j$. These weights are otherwise null. While $w_{i j}, \sigma_{i j}^{+}$and $\sigma_{i j}^{-}$are as in (4), $\delta_{i j \Pi \Phi}^{+}$and 
$\delta_{i j \Pi \Phi}^{-}$are respectively the largest and the smallest non-zero singular values of $\mathrm{D}_{\mathrm{ij} \pi \phi}$. Furthermore, $\gamma_{\Pi \Phi}$ is a parameter chosen to reflect the confidence one has in the constraint that two planes are parallel. In all our experiments, we have set $\gamma_{\Pi \Phi}=1$. Note that with two images and only one pair of parallel planes, 4 constraints are imposed on the intrinsic parameters of the camera which suffices in theory to calibrate a camera with no skew.

\section{Experiments}

We provide here some of the results obtained through extensive experiments using simulated data and two examples with real images.

\subsection{Simulated Data}

The goal of our experiments with simulated data was twofold: (1) assess the quality of the estimated 3D structure and that of the intrinsic parameters using both Mendonça/Cipolla's self-calibration and our method with parallel planes; (2) compare the results obtained with our method against those obtained using the stratified method [2]. Note that this stratified method uses plane parallelism to estimate the position of the plane at infinity and, unlike our method, it does not directly relate parallelism constraints to the intrinsic parameters. Using [2], an additional step for estimating the intrinsic parameters and another one for refining them have been used in our experiments.

All our simulations were carried out for various lengths of the image sequence and levels of noise added to image coordinates. For each sequence length and noise level, we have tested each method on 200 independent, randomly generated, sets of scenes and cameras. Each scene consisted of a randomly generated pair of parallel planes. 50 scene points scattered in a disc of unit-radius have been randomly generated for each plane. These points have been then projected onto a set of images each with a size of $512 \times 512$ pixels. The first plane was generated such that its disc was centered in the origin of the scene's reference frame. The second plane was generated parallel to the first one and at a mean distance of 0.5 units ( 0.25 standard deviation) from it. The cameras were generated at a mean distance of 2.5 units with 0.25 standard deviation from the center of the scene and roughly oriented towards the origin of the scene's reference frame. Camera parameters $f_{u}=f_{v}=800, s=0$ and $u_{0}=v_{0}=256$ were kept constant throughout the sequence. Zero-mean Gaussian noise with standard deviation in the range 0 to 2 pixels (with a step of 0.25 pixel) was added to the pixel coordinates. The intrinsic parameters estimated by each method along with the ground truth overall scale of the scene have been used to recover the 3D Euclidean structure by triangulation. The 3D Root-Mean-Square (RMS) error of the reconstructed points was calculated. Only simulations leading to a 3D RMS error $<0.65$ were considered as successful. When using the stratified [2], the DIAC was also required to pass the positive-definiteness test. For each noise level and sequence length, the average 3D RMS error and the relative RMS 
error on the estimated intrinsic parameters were calculated over the successful trials out of the 200 independent runs. The skew factor $s$ was always assumed to be known. Following this account, we have carried out two independent sets of experiments:

- the first set involved our method with parallel planes versus Mendonça/ Cipolla's self-calibration method. For each simulated scene and cameras, optimization was started from the same initial guess of the parameters using both the scene-constraints-free objective (4) and the one incorporating plane parallelism (13). At each trial, we have considered two initial values of the intrinsic parameters to start optimization from:

(a) the true noise-free values of the intrinsic parameters,

(b) randomly chosen parameters in which the values of $f_{u}$ and $f_{v}$ were picked in the range 1 to 2000 while those of the coordinates $u_{0}$ and $v_{0}$ were taken in a $200 \times 200$ pixels area centered at image coordinates $(256,256)$.

- the second set of experiments involved the method presented in this paper versus the stratified method [2. Optimization of (13) was randomly started as described in (b) for the first set of experiments. The method in [2] does not require initialization: it proceeds by identifying the plane at infinity through solving sets of quartic equations.

The results obtained using our method with one pair of parallel planes and two images (for various levels of noise) are summarized in Fig. 1], The average 3D RMS errors, Fig. 1f(a), and the relative RMS errors (in \%) on the intrinsic parameters, Fig. 1-(a), were calculated after initializing our objective function (13) from the true noise-free intrinsic parameters. These errors have been calculated over the successful trials (i.e. 3D RMS error $<0.65$ ) whose rate is given in Fig. 1 . (c). On the other hand Fig. 1 1 (d) provides the convergence rate (convergence to the correct parameters) of the optimization when the latter is randomly started. These results show that the errors obtained are fair considering only two images have been used. However, a good initial guess is definitely required in such minimal situation even in the absence (or low levels) of noise.

The results of similar experiments with 3 images, using both our method with one pair of parallel planes and the Mendonça-Cipolla's, are summarized in Fig. 2, It is clear from these results that incorporating plane parallelism constraints plays a very important role in improving not only the quality of the reconstruction (Fig. 2.(a)) and the parameters (Fig. 2.(b) for our method and Fig. 2. (c) for Mendonça and Cipolla's) but also the convergence of the algorithm. It can bee seen in Fig. 2. (e) that our method has converged (from random start) in $85 \%$ of the trials with 2 pixels of noise when considering plane parallelism while the method not considering scene constraints has barely converged to the right solution in only $50 \%$ of the cases.

The results with varying sequence lengths (2 to 8 images) and 1.25 pixels of noise are reported in Fig. 3. For instance, Fig. 3. (a) shows that the quality of the $3 \mathrm{D}$ reconstruction remains in favor of using scene constraints even when 8 images are used. The same conclusion can be drawn with regards to the intrinsic parameters Fig. 3.(b) (our method) versus Fig. 3. (c) (Mendonça-Cipolla's). The 


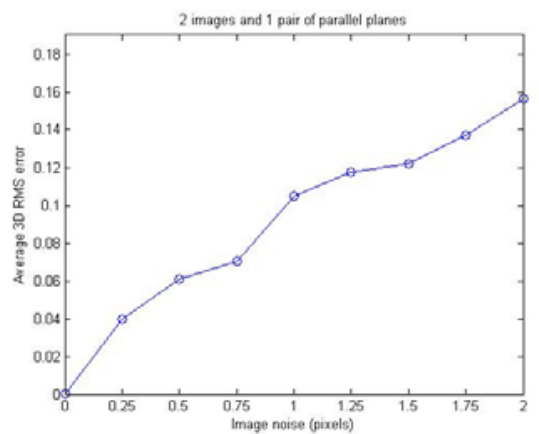

(a) $3 \mathrm{D}$ errors.

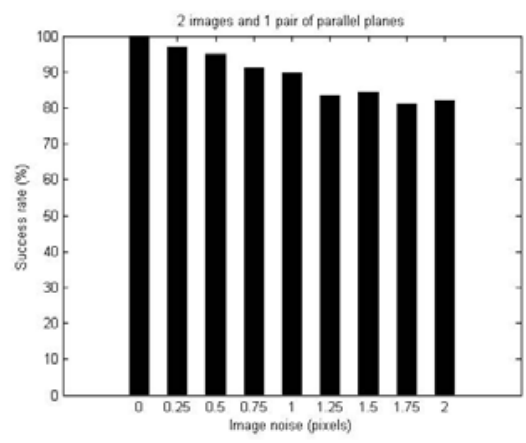

(c) Started from true int. param.

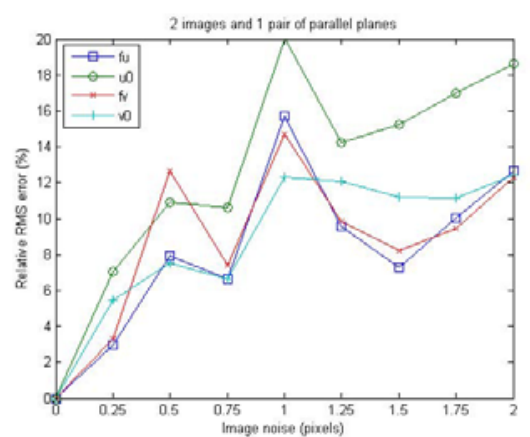

(b) Int. param. errors

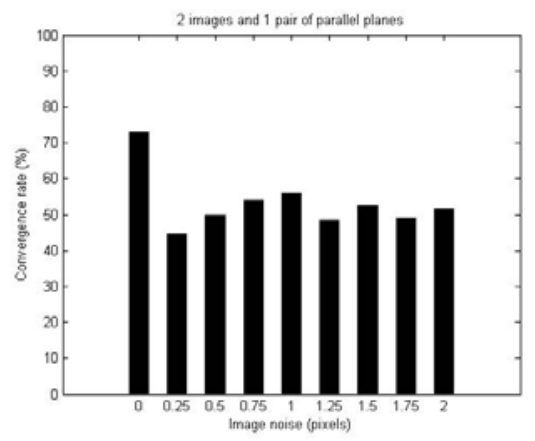

(d) Random start.

Fig. 1. Our method (1 pair of parallel planes): 2 images

convergence rates of the two methods are the same when using 6 or more images (Fig. 3.(d) and Fig. 3-(e)).

For comparison, the results obtained with the method presented here and those obtained with the stratified method 2 are reported in Fig. 4. Note that both methods use plane parallelism. The quality of the $3 \mathrm{D}$ reconstruction using (13) is better than the stratified method although the latter is based on a set of consistent projection matrices (usually leading to better results in sceneconstraints-free methods). This may be due to the fact that the stratified method requires this extra-calculation of a consistent set of projective camera matrices in which, unlike when considering the images pairwise, errors or noise in one image might propagate to the entire set of camera matrices. Note that in these experiments, optimization of (13) has been randomly initialized and the convergence rates are given in Fig. 44(b). For low levels of noise (typically $<0.75$ pixel), the stratified method converges slightly more often than (13) to the right solution. However, for higher levels of noise (typically $\geq 0.75$ pixel) our method has a better convergence rate. This can be explained by the fact that the stratified method finds it more difficult to single out the plane at infinity with the increasing noise. In addition, the step leading to an estimate of the intrinsic parameters from the plane at infinity becomes more sensitive to image noise. 


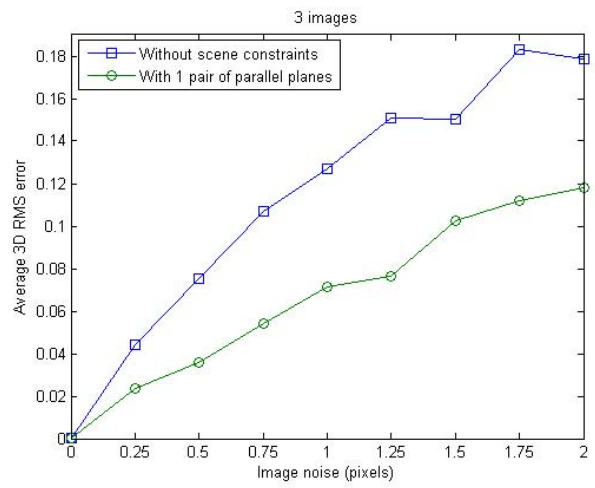

(a) $3 \mathrm{D}$ errors.

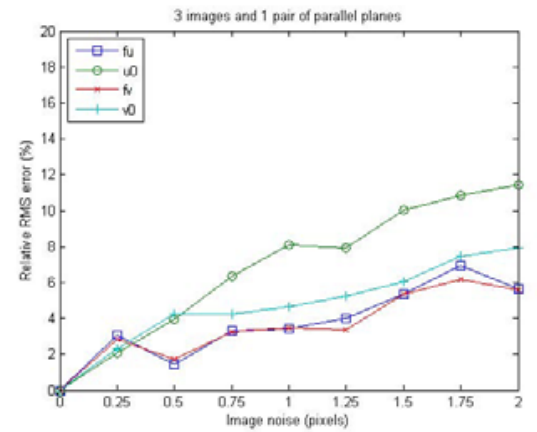

(b) Int. param. errors (our method).

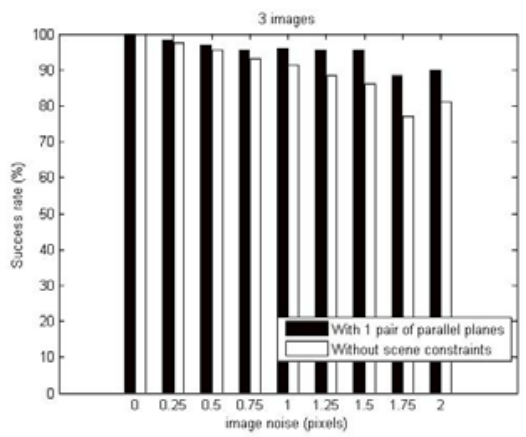

(d) Started from true int. param.

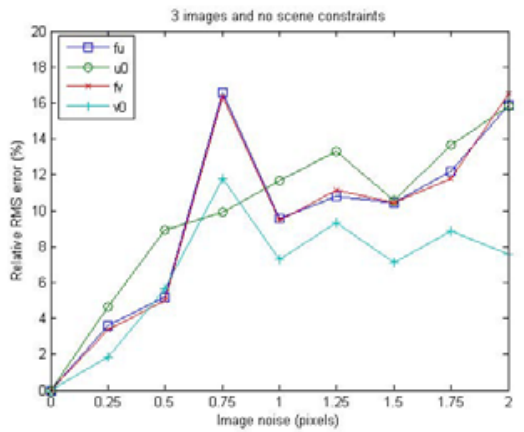

(c) Int. param. errors (Mendonça-Cipolla).

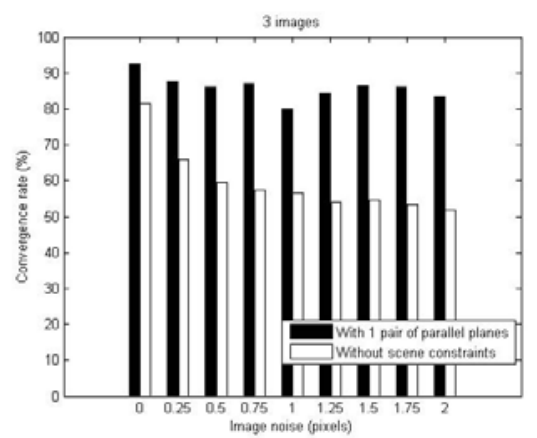

(e) Random start.

Fig. 2. Our method (1 pair of parallel planes) versus Mendonça-Cipolla's (without scene constraints): 3 images 


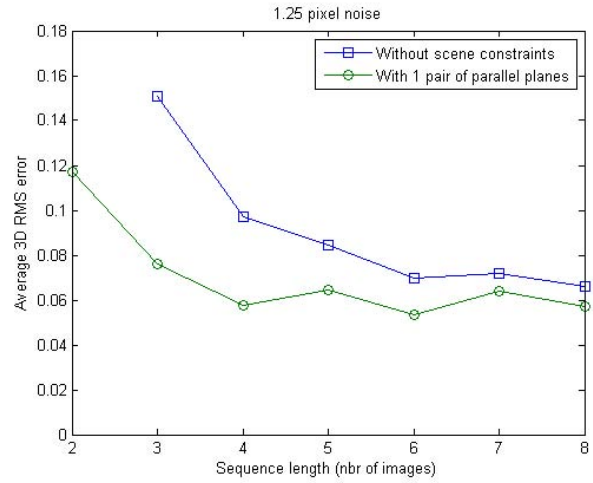

(a) $3 \mathrm{D}$ errors.

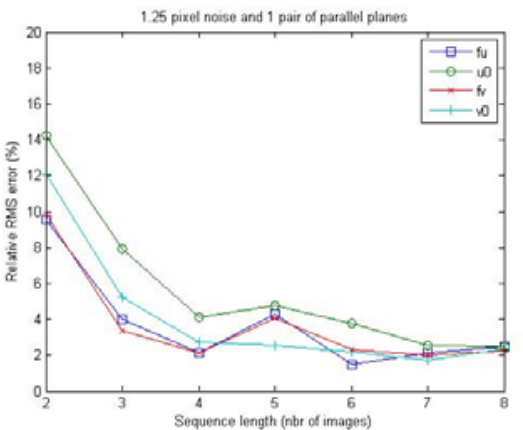

(b) Int. param. errors (our method).

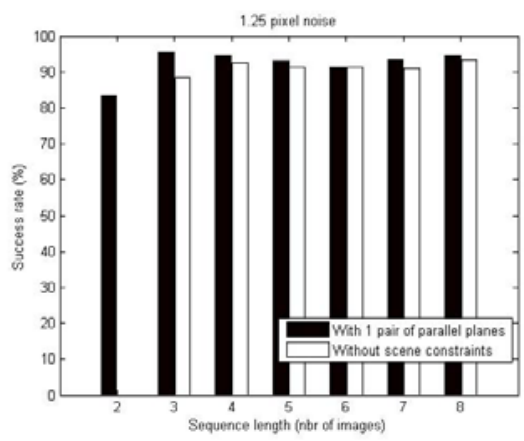

(d) Started from true int. param.

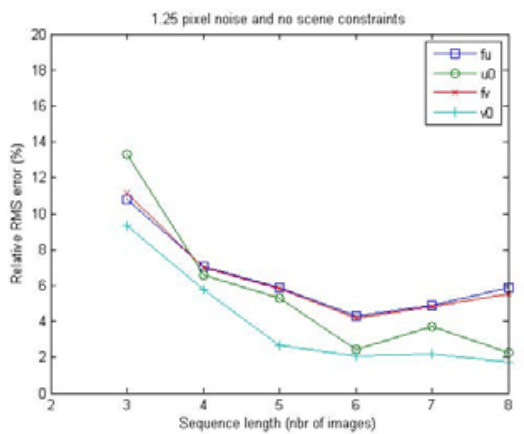

(c) Int. param. errors (Mendonça-Cipolla).

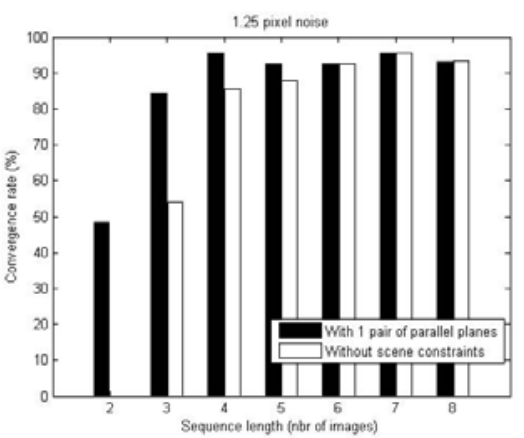

(e) Random start.

Fig. 3. Our method (1 pair of parallel planes) versus Mendonça-Cipolla's (without scene constraints): varying sequence lengths and 1.25 pixel noise 


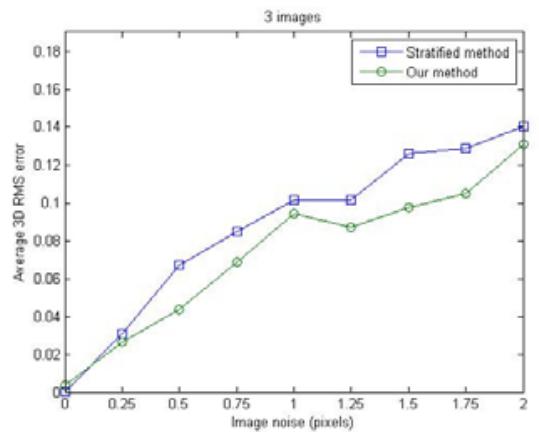

(a) $3 \mathrm{D}$ errors.

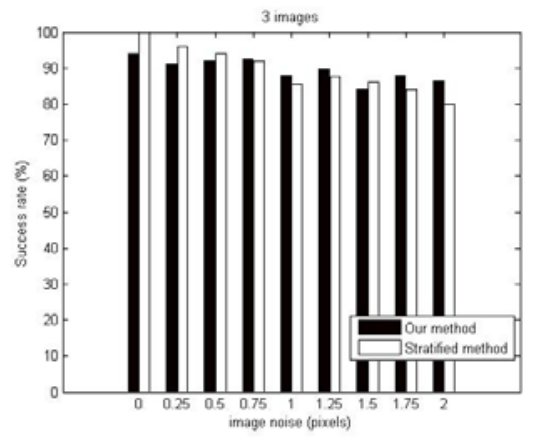

(b) Convergence rate.

Fig. 4. Our method (randomly started) vs. stratified method [2]: 3 images and 1 pair of parallel planes

\subsection{Real Images}

We have successfully tested our method on numerous real scenes of which we present here two examples: the "Patterns" scene, Fig. 5. and the "Desk" scene, Fig. 6. These images have been captured with a low-end Sony Cyber-shot DSCS930. Only 2 images have been used in each example. The 48 points marked in Fig. 5 (middle) where matched across the two images of the "Patterns" scene
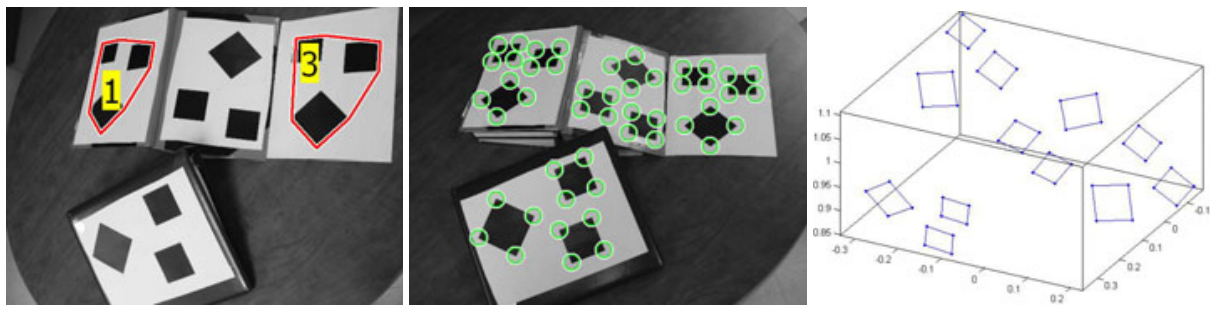

Fig. 5. The two images of the "Patterns" scene and its metric $3 \mathrm{D}$ reconstruction
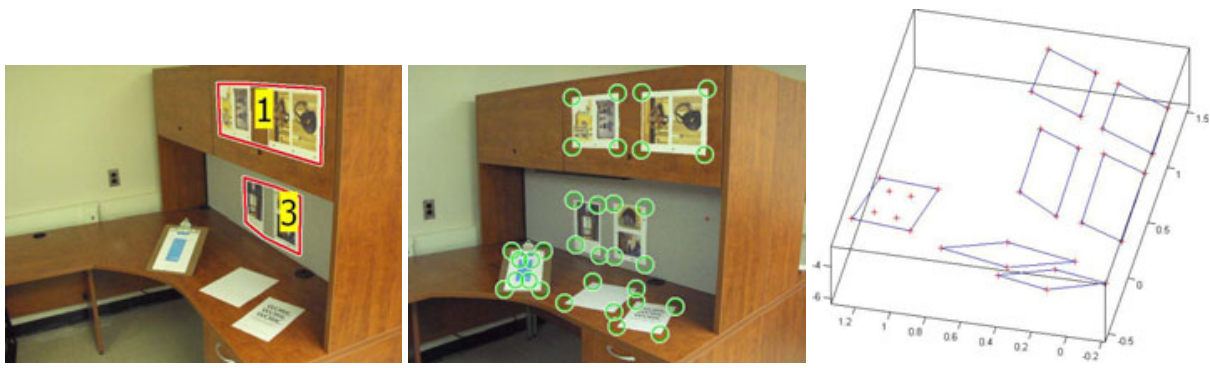

Fig. 6. The two images of the "Desk" scene and its metric 3D reconstruction 
while only the 28 points marked in Fig. 6 (middle) were matched across the images of the "Desk" scene. The planes labeled 1 and 3 in the left-hand side images in each figure were considered parallel. The recovered 3D metric structures of both scenes are given in Figs. 5 and 6] (right). The relative errors on the line segments ratios in both reconstructed scenes were found in the range $10 \%-18 \%$.

\section{Conclusion}

We have proposed a new method that combines the self-calibration constraints on the essential matrix with plane parallelism constraints. The method allows the calibration of a camera from point correspondences as soon as two images are available (instead of three in the absence of scene constraints) and considers each pair of images independently. Our experiments have demonstrated that (1) the scene can be reconstructed using our method with a good accuracy even when using few images and high levels of image noise; (2) scene constraints contribute to significantly improve the quality of the $3 \mathrm{D}$ reconstruction and convergence in comparison with the works in [2] and [7].

\section{References}

1. Fusiello, A.: A New Autocalibration Algorithm: Experimental Evaluation. In: Skarbek, W. (ed.) CAIP 2001. LNCS, vol. 2124, pp. 717-724. Springer, Heidelberg (2001)

2. Habed, A., Amintabar, A., Boufama, B.: Affine camera calibration from homographies of parallel planes. In: Proceedings of the IEEE International Conference on Image Processing, pp. 4249-4252 (2010)

3. Hartley, R.I., Zisserman, A.: Multiple View Geometry in Computer Vision, 2nd edn. Cambridge University Press (2004) ISBN: 0521540518

4. Huang, T.S., Faugeras, O.D.: Some properties of the e matrix in two-view motion estimation. IEEE Transactions on Pattern Analysis and Machine Intelligence 11(12), 1310-1312 (1989)

5. Huynh, D.Q., Heyden, A.: Scene point constraints in camera auto-calibration: An implementational perspective. Image and Vision Computing 23(8), 747-760 (2005)

6. Liebowitz, D., Zisserman, A.: Combining scene and auto-calibration constraints. In: Proceedings of the 7th International Conference on Computer Vision, Kerkyra, Greece, pp. 293-300 (1999)

7. Mendonça, P.R.S., Cipolla, R.: A simple technique for self-calibration. In: Proceedings of the Conference on Computer Vision and Pattern Recognition, Fort Collins, Colorado, USA, vol. 1, pp. 500-505 (June 1999)

8. Pollefeys, M., Van Gool, L.: Stratified self-calibration with the modulus constraint. IEEE Transactions on Pattern Analysis and Machine Intelligence 21(8), 707-724 (1999) 